\title{
CONTRIBUCIÓN AL ANÁLISIS NIVOMÉTRICO DEL PIRINEO ORIENTAL: LA MOLINA, PERIODO 1956-1996
}

\author{
Ferran SALVADOR FRANCH ${ }^{1,2}$, Gabriel SALVÀ VILLOSLADA ${ }^{2}$, \\ Francesc VILAR BONET ${ }^{2}$, Carles GARCÍA SELLÉS ${ }^{3}$ \\ ${ }^{\prime}$ Departament de Geografia Física i A.G.R. Universitat de Barcelona. \\ ${ }^{2}$ Servei de Gestió i Evolució del Paisatge. Universitat de Barcelona. \\ ${ }^{3}$ Institut Cartogràfic i Geologic de Catalunya. \\ fsalvador@ub.edu, gabrisv@gmail.com, photo.dng@gmail.com, carles.garcia@icgc.cat
}

\section{RESUMEN}

A partir de los datos diarios de precipitación, permanencia y espesor de nieve, se han calculado los valores más significativos de nivosidad e innivación en La Molina ( $1.703 \mathrm{~m}$, extremo oriental del Pirineo), durante 38 temporadas nivales (de noviembre a mayo, de 1956/57 a 1995/96). Su rasgo más destacado es la acusada irregularidad de todas las variables nivoclimáticas estudiadas, tanto las relacionadas con la cuantía y frecuencia de la precipitación nivosa, como las relacionadas con la permanencia y espesor del manto de nieve.

Palabras clave: nivoclimatología, nivosidad, innivación, La Molina, Pirineo oriental.

\begin{abstract}
Daily snow data in La Molina (1.703 m asl, eastern Pyrenees) is analysed during 38 nival seasons (November to May; 1956/57 to 1995/96), from which the most significant values of snowfall and snow cover are calculated. It should be pointed the accused irregularity of all climatic characteristics studied: the amount and frequency of snow precipitation, as well as the persistence and thickness of the mantle of snow.
\end{abstract}

Key words: nivoclimatology, snowfall, snow cover, La Molina, Eastern Pyrenees.

\section{INTRODUCCIÓN}

Con el propósito de seguir contribuyendo a la caracterización nivoclimática del Pirineo oriental, abordada ya en trabajos anteriores (Salvador Franch, 1985; García Sellés y Salvador Franch, 1994; Vilar Bonet y Salvador Franch, 2001; Salvador Franch et al., 2014), se presenta ahora una sucinta aproximación al análisis de los datos nivométricos diarios disponibles del observatorio meteorológico de La Molina situado a $1.703 \mathrm{~m}$ de altitud (38 períodos invernales, de noviembre a mayo, entre 1956-57 y 1995-96). Esta serie es complementaria de la disponible en la cercana Núria (1.970 m; 1985-86 a 2012-13), recientemente estudiada (Salvador Franch et al., 2014), entre ambas permiten describir la nivometría de este sector pirenaico desde mitad del pasado siglo.

Aun siendo bien conocidos los rasgos fundamentales del clima pirenaico, y en nuestro caso, de su extremo oriental (Xercavins, 1981; Martín Vide, 1985; entre 
otros), sorprende la escasa atención recibida por la nieve, a pesar de su indiscutible interés, tanto climático como también turístico y deportivo.

\section{2. ÁREA DE ESTUDIO}

El valle de La Molina se ubica en la ladera noreste de la Tosa d'Alp (2.536 m) y norte del Puigllançada $(2.409 \mathrm{~m})$ que, a su vez, forman parte de la alineación montañosa Cadí-Puigmal que delimita por el sur la fosa tectónica de la Cerdanya (Vila, 1926), vertiendo aguas al alto valle del Segre, aún de marcada influencia mediterránea, tanto climática como biogeográfica (Xercavins, 1981) (figura1). La llegada en 1923 del ferrocarril transpirenaico a La Molina (línea Barcelona-Puigcerdà) determinó que este valle tuviera un papel pionero en el inicio y posterior desarrollo de los deportes de invierno en la cordillera pirenaica (Guilera, 1931; Roger, 2006).
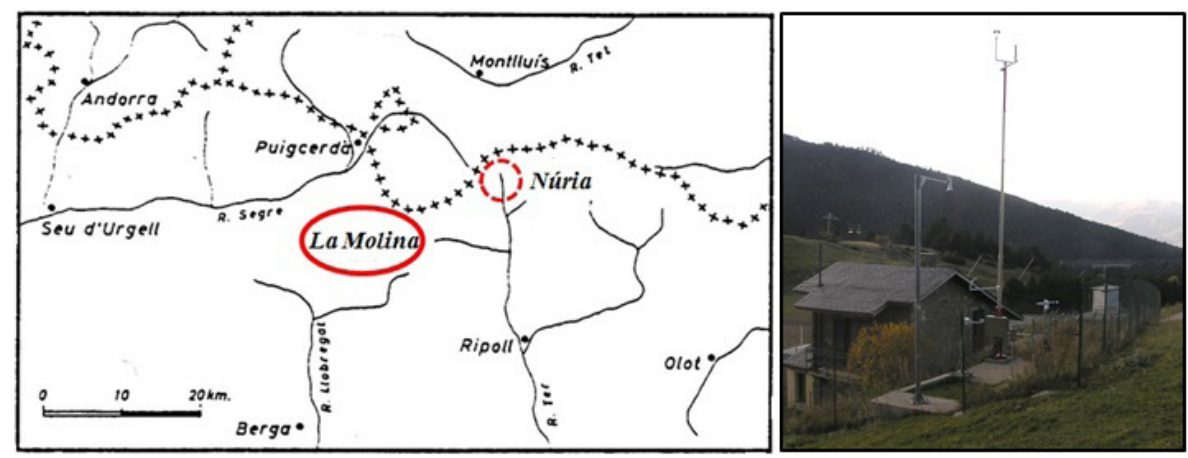

Fig. 1: Situación de La Molina en el Pirineo Oriental y observatorio de referencia.

Las observaciones meteorológicas en La Molina se inician en 1927 (cota $1.500 \mathrm{~m}$ ) hasta interrumpirse en 1936, retomándose, ya en su emplazamiento actual (Font Canaleta, $1.703 \mathrm{~m}$ ), a principio de la década de los 50 . La serie continua y con datos de nieve, comienza en 1955. Durante varias décadas este observatorio formó parte de la red principal del antiguo SMN (Servicio Meteorológico Nacional), más tarde INM (Instituto Nacional de Meteorología), actual AEMET. Junto con el Turó de l'Home (Montseny, $1.706 \mathrm{~m}$ ), fueron los dos únicos observatorios de primer orden, en Catalunya, situados en áreas de montaña. A mitad de los 90, la falta de observador permanente provocó la interrupción temporal de la serie y, más tarde, la instalación de una estación automática. El clima de La Molina es de alta montaña mediterránea, con precipitación relativamente abundante $(\sim 1.200 \mathrm{~mm})$ y régimen OVPI de transición al VOPI, siendo por tanto el periodo nival el más deficitario (Martín Vide, 1985). Por su altitud, las temperaturas son moderadamente frías en invierno, con \pm 150 días de helada y suaves el resto del año (SMC, 2008).

La pluviometría media anual se sitúa en $1.190 \mathrm{~mm}$, el $54 \%$ durante el periodo de noviembre a mayo, pero solo el $32 \%$ de diciembre a abril. La distribución de los valores anuales presenta una moderada irregularidad (CV 21,8\%), sus máximos extremos casi triplican a los mínimos anuales, pero los máximos registrados en el periodo diciembre-abril pueden ser ocho veces superiores a los mínimos del mismo periodo 
(CV 46,5\%). La temperatura media anual es de $6,0^{\circ} \mathrm{C}$, situándose en $1,9^{\circ} \mathrm{C}$ durante el período de precipitación nival (noviembre-mayo) (tabla 1, figura 2).

\begin{tabular}{|c|cc|ccc|}
\hline & \multicolumn{2}{|c|}{ Temperatura } & \multicolumn{3}{c|}{ Precipitación } \\
\cline { 2 - 6 } & $\begin{array}{c}\text { T media anual } \\
(\mathrm{St}-\mathrm{Ag}) \\
\left({ }^{\circ} \mathrm{C}\right)\end{array}$ & $\begin{array}{c}\text { T media pn } \\
(\mathrm{Nv}-\mathrm{My}) \\
\left({ }^{\circ} \mathrm{C}\right)\end{array}$ & $\begin{array}{c}\text { Pt anual } \\
(\mathrm{St}-\mathrm{Ag}) \\
(\mathrm{mm})\end{array}$ & $\begin{array}{c}\text { Pt pn } \\
(\mathrm{Nv}-\mathrm{My}) \\
(\mathrm{mm})\end{array}$ & $\begin{array}{c}\text { Pt pnr } \\
(\mathrm{Dc}-\mathrm{Ab}) \\
(\mathrm{mm})\end{array}$ \\
\hline media & $\mathbf{6 , 0}$ & $\mathbf{1 , 9}$ & $\mathbf{1 . 1 8 9 , 8}$ & $\mathbf{6 4 2 , 4}$ & $\mathbf{3 7 8 , 0}$ \\
máx. & $7,7(88-89,89-90)$ & $4,1(89-90)$ & $1.718,5(63-64)$ & $1.026,3(58-59)$ & $899,4(58-59)$ \\
mín. & $4,1(71-72)$ & $-0,7(62-63)$ & $613,8(85-86)$ & $335,9(93-94)$ & $102,8(84-85)$ \\
C.V. & 16,3 & 61,0 & 21,8 & 29,8 & 46,5 \\
\hline
\end{tabular}

Pt: Precipitación total indiferenciada, expresada en forma líquida.

pn: periodo nival extenso, de noviembre a mayo.

pnr: periodo nival reducido, de diciembre a abril. (entre paréntesis): fecha de los valores extremos

Tabla 1: Valores termopluviométricos de La Molina, periodo 1956-57 a 1995-96

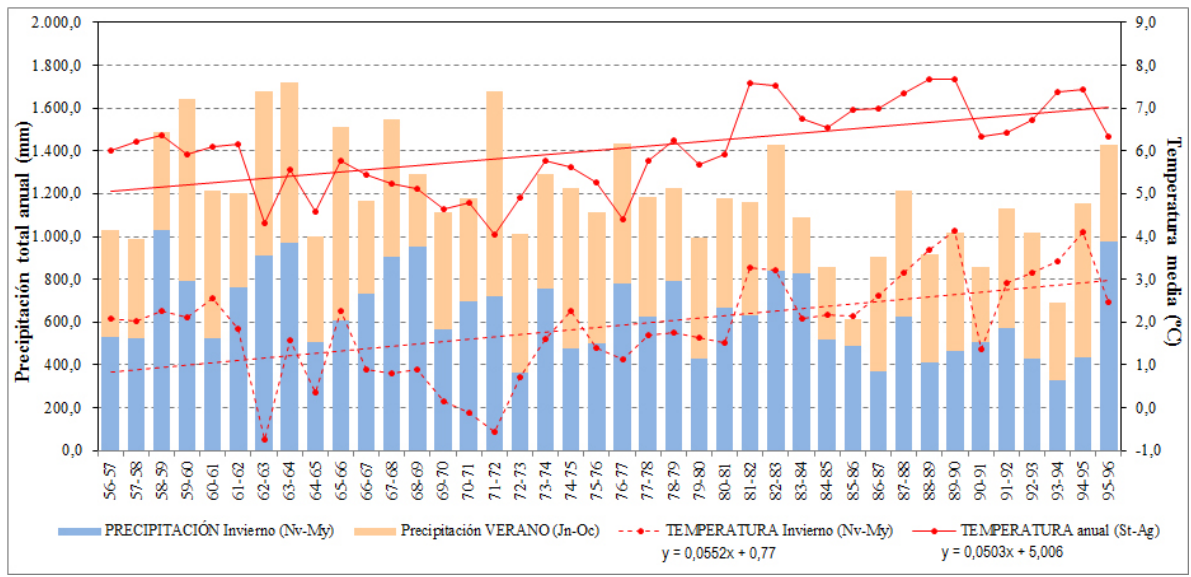

Fig. 2: Evolución termopluviométrica de La Molina. Periodo 1956-57 a 1995-96.

\section{MATERIALES Y MÉTODOS}

Los índices nivométricos que se presentan, se han obtenido a partir del tratamiento de los datos diarios, procedentes de los cuadernos de observación originales, de: precipitación líquida o sólida (expresada en $\mathrm{mm}$ ), espesor diario de nieve recién caída (en $\mathrm{cm}$ ) y espesor total del manto nival acumulado (en $\mathrm{cm}$ ). Debido a la falta de información de los años 81-82 y 82-83 en la cota de referencia, en los perfiles de innivación de la figura $3 \mathrm{~b}$ se han incluido (solo a efectos de representación gráfica, en trama de puntos) los espesores obtenidos por la Estación de Esquí de La Molina durante 8 temporadas invernales (81-82 a 88-89) en la cota 2.050 m (La Molina-Costa Rasa). 

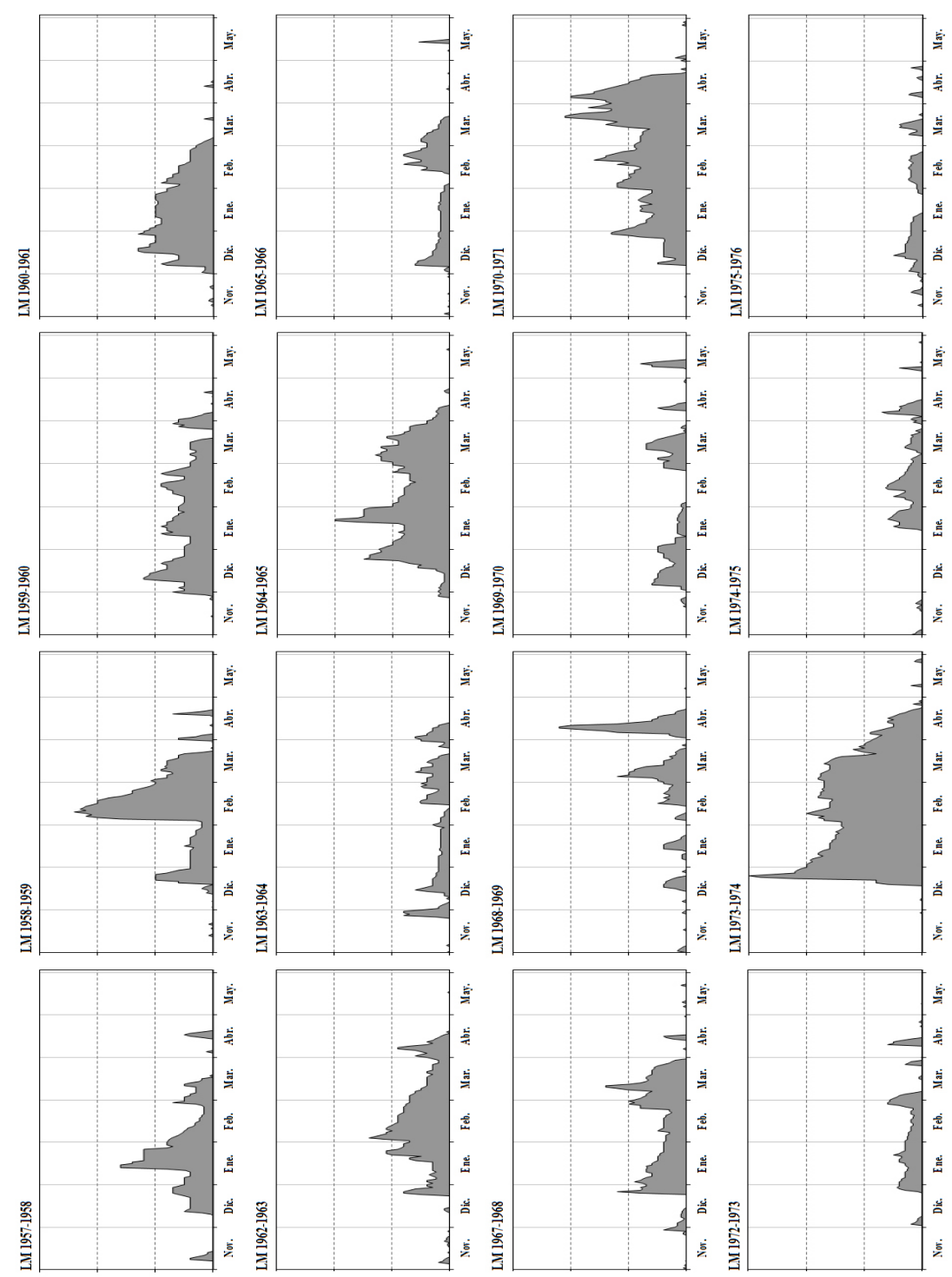

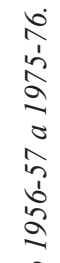
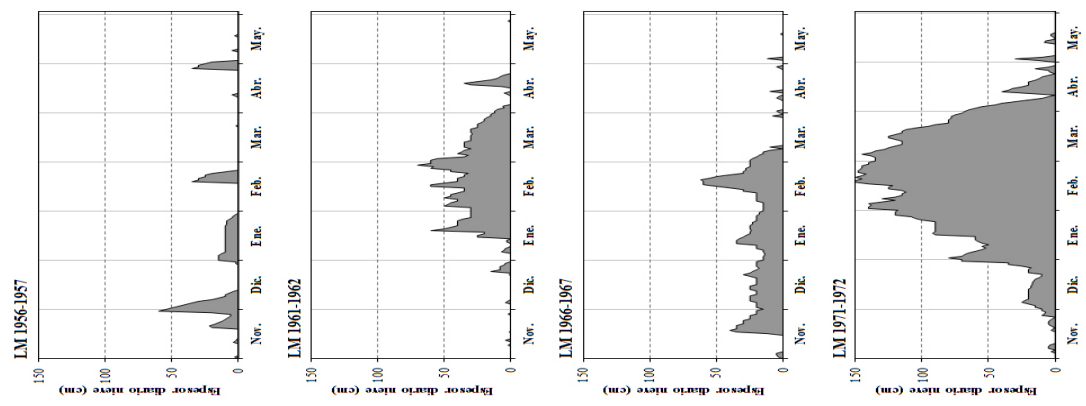

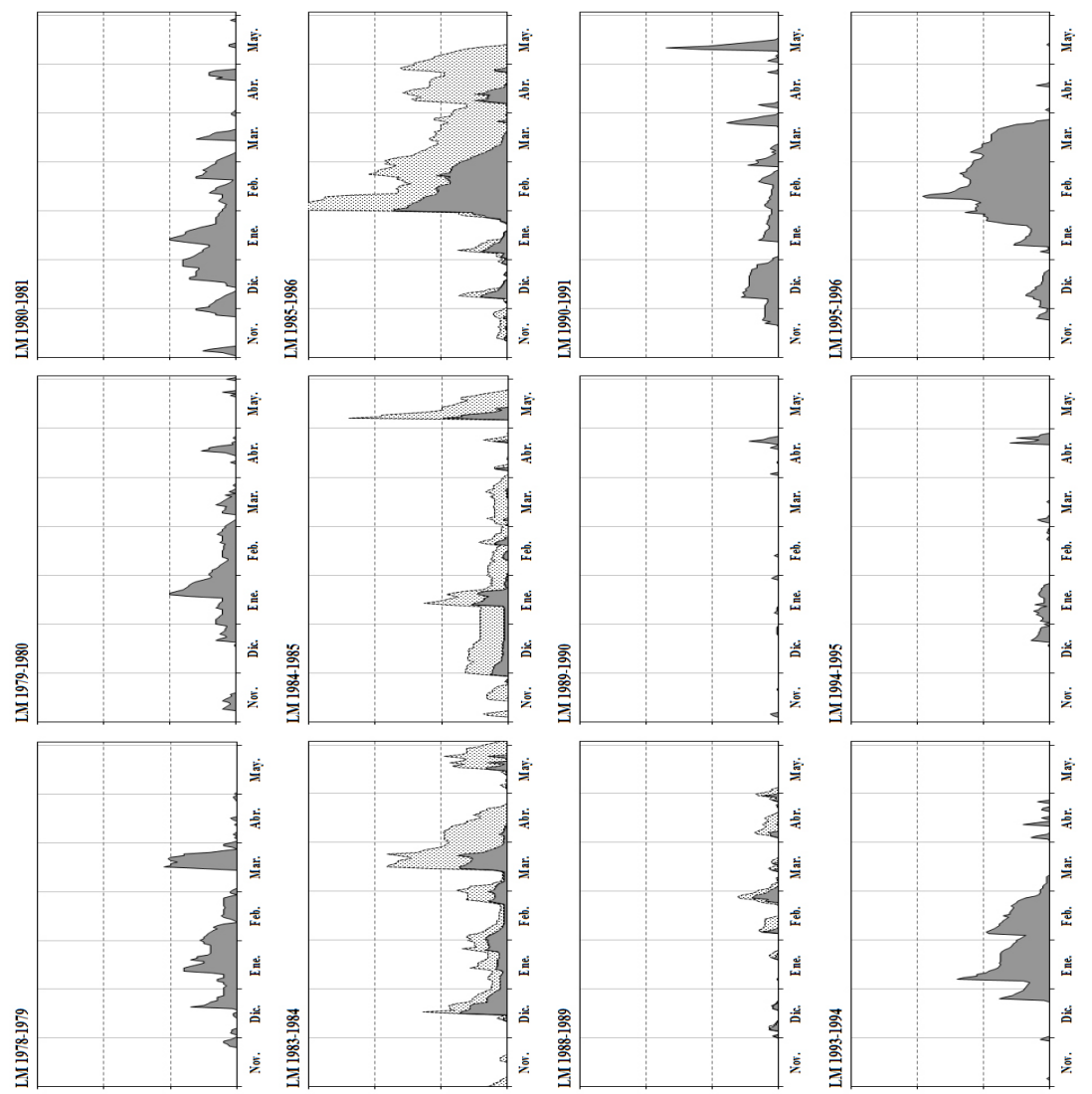

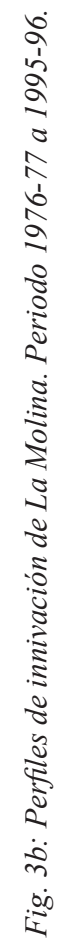
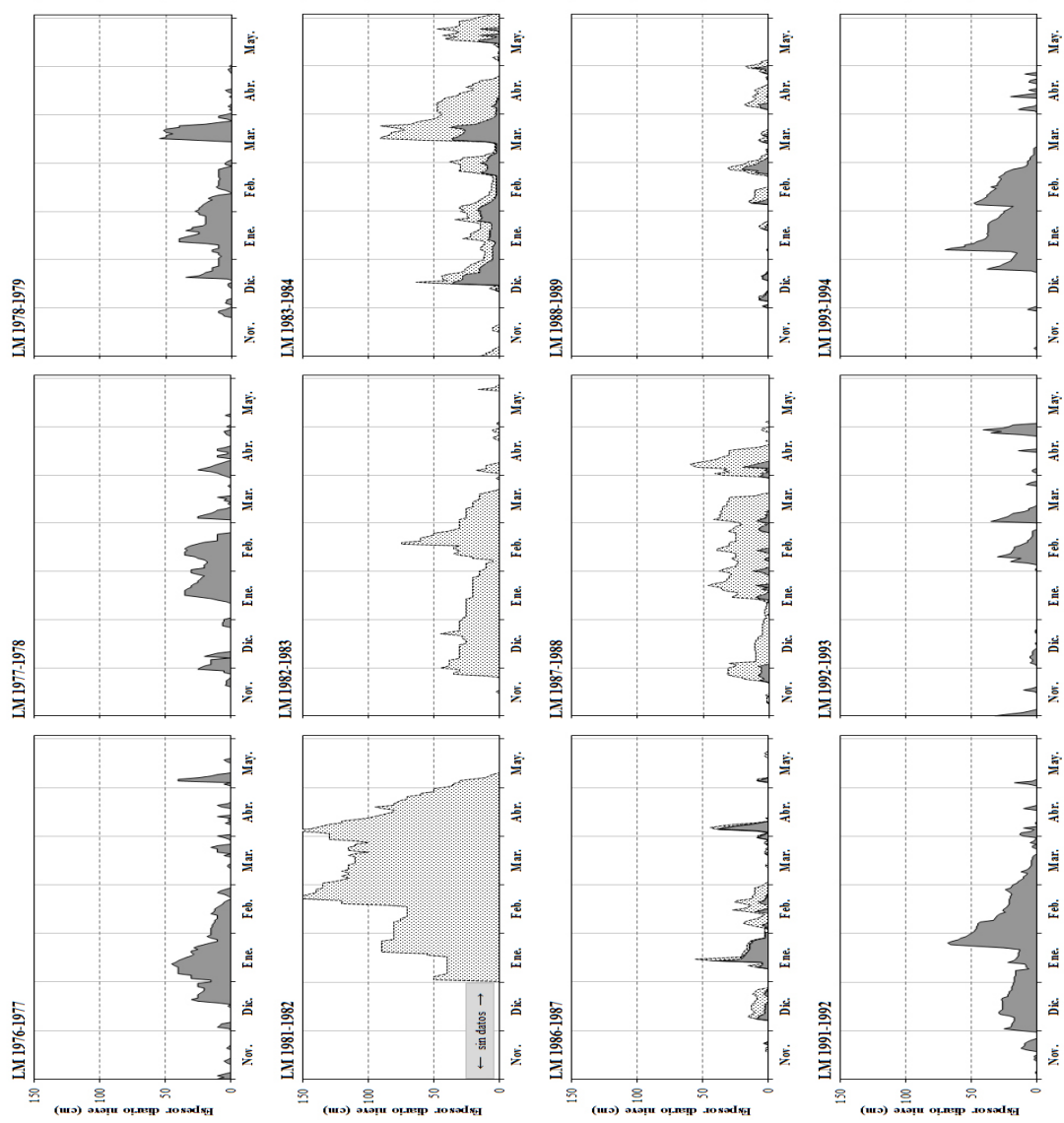


\section{RESULTADOS}

Con objeto de facilitar la comparación con los valores nivométricos anteriormente obtenidos en Núria (Salvador Franch et al., 2014) se han seguido, en lo posible, salvo mención específica (apartado 4.2 y tabla 2), los mismos procedimientos de tratamiento de datos y de presentación de resultados.

\subsection{Perfiles de innivación}

La observación de los 40 perfiles de innivación obtenidos (figuras 3a y b) y, más adelante, el tratamiento estadístico de sus datos, pone sin duda de manifiesto, igual que en Núria, la característica más destacada del comportamiento nival de este sector pirenaico, es decir, su muy acusada irregularidad, que se manifiesta en la variabilidad de las fechas de inicio y final del periodo de innivación, en la continuidad o fragmentación de la presencia del manto, o en los valores del espesor acumulado del manto. Los años de innivación extremadamente deficitaria (p.ej. 88-89, 89-90, 94-95) se alternan con otros de innivación escasa o mediana, u otros con presencia nival abundante o muy abundante (p.ej. 64-65, 70-71, 71-72, 73-74, 81-82, ...) sin periodicidad aparente. En conjunto, sin embargo, se aprecia una clara tendencia a la disminución de los valores nivométricos de referencia, en el área y periodo de estudio (figuras 4, 5 y 6), tanto en lo que se refiere a cantidades de precipitación, días de suelo cubierto de nieve, como al espesor acumulado. También, como en Núria y en otros enclaves pirenaicos y cordilleras peninsulares, los buenos y malos años en cuanto a presencia nival pueden sucederse (p.ej. 94-95 y 95-96) sin formar parte de ciclos plurianuales. Destaca así mismo, la frecuencia de algunos rasgos característicos como son las nevadas de cierta magnitud a final de temporada, desconectadas ya del manto invernal (p.ej. abril del 69, mayos del 70, 85 y 91, entre otros), o la elevada intensidad de algunos episodios (p.ej. febreros del 59 y 86, diciembre del 73, diversos eneros).

\subsection{Nivosidad}

La nieve caída (nivosidad) anual media es modesta $(251 \mathrm{~cm})$, con máximos que superan los $500 \mathrm{~cm}$ (71-72) y un mínimo excepcional de solo $56 \mathrm{~cm}$ en la temporada 89-90, dando como resultado un CV muy elevado, del 36\%. La mayor parte de la nivosidad (el 86\%) se da entre diciembre y abril. En conjunto, es fruto de un promedio de 36 días de precipitación de nieve con un CV del 28\%, registrándose un máximo de 71 días (71-72) y un mínimo de 16 (89-90). A partir de los años 80 se observa una tendencia a la disminución de la cantidad $(\mathrm{cm})$ y frecuencia (días) de la precipitación en forma de nieve (figuras 4 y 5), al mismo tiempo que se produce un incremento de las temperaturas medias (figura 2).

En La Molina, a diferencia de Núria, el cálculo del coeficiente nivométrico $(\mathrm{CN}$, relación entre la precipitación total y la caída en forma de nieve) ha podido realizarse de forma precisa a partir de los datos diarios del equivalente líquido de la nieve recién caída. Así se obtiene un CN anual medio del 33\% (CV: 30\%), incrementándose, lógicamente, el $\mathrm{CN}$ según se reduce el periodo considerado, a la vez que disminuye el CV (CN: 60\%, CV: 24\%, de noviembre a mayo; CN: 81\%, CV:16\%, de diciembre a abril) indicando, no obstante, como ya se vio en Núria, que se produce un volumen 
de precipitación líquida apreciable (entre el 40 y el 19\%) durante el periodo invernal y nival (tabla 2).

La intensidad de la precipitación de nieve (cantidad máxima caída en 24 horas), presenta una variabilidad muy elevada (CV: $38 \%$ ), con un promedio de $36 \mathrm{~cm}$, aunque oscila entre mínimos de sólo $10 \mathrm{~cm}$ (otra vez 89-90) y 2 máximos de $70 \mathrm{~cm}$ registrados en febrero del 59 y diciembre del 73, ambos causados por una situación persistente de precipitaciones de origen mediterráneo (tabla 2, figuras 3a y 4).

\begin{tabular}{|c|ccccc|c|c|}
\hline & \multicolumn{3}{|l|}{ Cantidad de nieve caída } & & & Frecuen. & Intensid. \\
\cline { 2 - 8 } & $\begin{array}{c}\text { Pn pn } \\
(\mathrm{Nv}-\mathrm{My}) \\
(\mathrm{cm})\end{array}$ & $\begin{array}{c}\text { Pn pnr } \\
(\mathrm{Dc}-\mathrm{Ab}) \\
(\mathrm{cm})\end{array}$ & $\begin{array}{c}\text { CNanual } \\
(\mathrm{St}-\mathrm{Ag}) \\
(\%)\end{array}$ & $\begin{array}{c}\text { CN pn } \\
(\mathrm{Nv}-\mathrm{My}) \\
(\%)\end{array}$ & $\begin{array}{c}\text { CN pnr } \\
(\mathrm{Dc}-\mathrm{Ab}) \\
(\%)\end{array}$ & $\begin{array}{c}\text { D Pn } \\
(\mathrm{Nv}-\mathrm{My}) \\
(\text { días })\end{array}$ & $\begin{array}{c}\text { Pn máx. } \\
\text { en 24h } \\
(\mathrm{cm})\end{array}$ \\
\hline media & $\mathbf{2 5 1}(203)$ & $\mathbf{2 1 6}(171)$ & $\mathbf{3 2 , 5}$ & $\mathbf{6 0 , 4}$ & $\mathbf{8 0 , 6}$ & $\mathbf{3 6}(27)$ & $\mathbf{3 6}$ \\
máx. & $557(392)$ & $469(339)$ & 55,0 & 92,2 & 99,5 & $71(48)$ & 70 \\
mín. & $56(54)$ & $49(47)$ & 11,0 & 29,2 & 45,4 & $16(12)$ & 10 \\
C.V. & $36,1(33,0)$ & $40,1(37,8)$ & 30,1 & 24,0 & 15,9 & $28,4(26,7)$ & 38,2 \\
\hline
\end{tabular}

Pn: Precipitación en forma de nieve.

pn: periodo nival extenso, de noviembre a mayo.

pnr: periodo nival reducido, de diciembre a abril.

$C N$ : Coeficiente nivométrico.

D Pn: Días de precipitación en forma de nieve.

(entre paréntesis): valor obtenido con el método utilizado para Núria.

Tabla 2: Valores de nivosidad en La Molina, periodo 1956-57 a 1995-96.

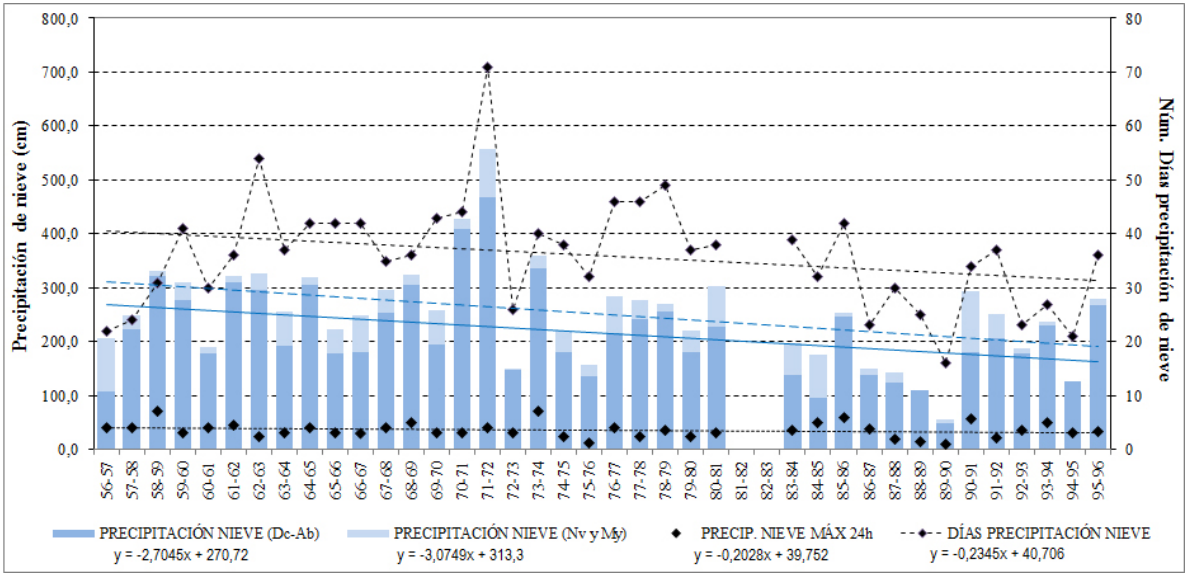

Fig. 4: Evolución de la nivosidad en La Molina. Periodo 1956-57 a 1995-96.

\subsection{Innivación}

El suelo permanece cubierto de nieve un promedio de 106 días al año, con un máximo registrado de 170 (71-72) y un mínimo de sólo 23 días (89-90), aunque en 
realidad a un número considerable de esos días les corresponde un espesor muy reducido. Por tanto, si consideramos únicamente los días con espesor $\geq$ a $10 \mathrm{~cm}$, los valores de permanencia se reducen significativamente, a un promedio de 74 días, con dos picos destacados en las temporadas 70-71 y 71-72, con 135 y 146 días respectivamente, y cuatro mínimos inferiores a 10 días a finales de la serie (87-88, 88-89, 89-90 y 94-95). Si además, consideramos solo el más prolongado de los periodos en que el manto nival fue de forma continua $\geq$ a $10 \mathrm{~cm}$, entonces obtenemos valores de permanencia estable y significativa de la nieve de 54 días en promedio, también con máximos en las temporadas 70-71 y 71-12, de 134 y 130 días respectivamente, mientras que en la temporada 89-90 se dio un mínimo excepcionalmente bajo de sólo 2 días con más de $10 \mathrm{~cm}$ de nieve, y precisamente ya a finales de abril. El CV de los valores de permanencia aumenta muy considerablemente a medida que su criterio de expresión se restringe, pasando del 28 al 73\% (tabla 3, figuras 5 y 6).

\begin{tabular}{|c|ccc|cc|}
\hline \multirow{2}{*}{} & \multicolumn{2}{|c|}{ Permanencia de la nieve en el suelo } & \multicolumn{2}{c|}{ Espesor de la nieve en el suelo } \\
\cline { 2 - 6 } & $\begin{array}{c}\text { Dns } \\
\text { (Nv-My) } \\
\text { (días) }\end{array}$ & $\begin{array}{c}\text { Dns } \\
\geq 10 \mathrm{~cm} \\
\text { (días) }\end{array}$ & $\begin{array}{c}\text { D máx. } \\
\text { mn } \geq 10 \mathrm{~cm} \\
(\text { días })\end{array}$ & $\begin{array}{c}\text { Espesor máx. } \\
\text { (Nv-My) } \\
(\mathrm{cm})\end{array}$ & $\begin{array}{c}\text { Espesor } \\
\text { medio* } \\
(\mathrm{Nv-My)} \\
(\mathrm{cm})\end{array}$ \\
\hline media & $\mathbf{1 0 6}$ & $\mathbf{7 4}$ & $\mathbf{5 4}$ & $\mathbf{6 3}$ & $\mathbf{2 1}$ \\
valor máx. & $170(71-72)$ & $146(71-72)$ & $134(70-71)$ & $150(71-72,73-74)$ & $67(73-74)$ \\
valor mín. & $23(89-90)$ & $2(89-90)$ & $2(89-90)$ & $20(87-88,88-89)$ & $4(89-90)$ \\
C.V. & 28,1 & 49,6 & 73,3 & 52,4 & 68,3 \\
\hline
\end{tabular}

Dns: Duración de la nieve cubriendo el suelo.

D máx mnc: Duración máxima del periodo con presencia de manto nival continuo.

(*): en relación al número de días con nieve cubriendo el suelo. . (entre paréntesis): fecha de los valores extremos

Tabla 3: Valores de innivación en La Molina, periodo 1956-57 a 1995-96.

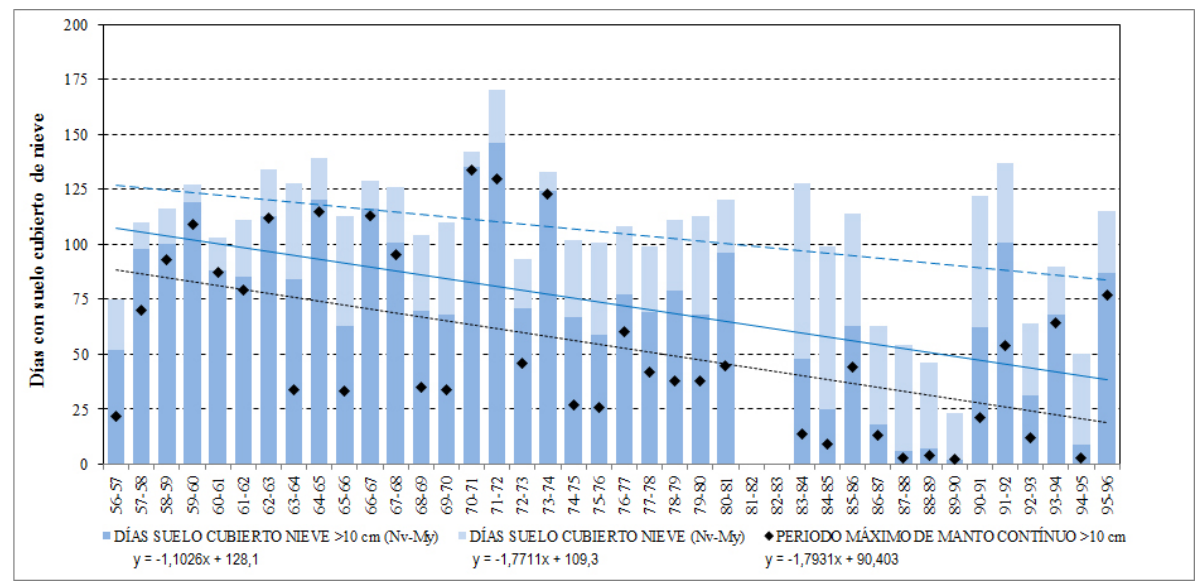

Fig. 5: Evolución de la innivación en La Molina. Periodo 1956-57 a 1995-96. 
El espesor máximo alcanzado por el manto de nieve ha sido de $150 \mathrm{~cm}$ (en 71-72 y 73-74), seguido de $120 \mathrm{~cm}$ (58-59) y $110 \mathrm{~cm}$ (68-69). Sin embargo, en 87-88, 8889 y $89-90$ sólo se alcanzaron máximos igual o ligeramente superiores a $20 \mathrm{~cm}$. Su promedio se sitúa en torno a los $63 \mathrm{~cm}$ (CV: 52\%). Por otro lado, el espesor medio del manto calculado en relación al número total de días con presencia de nieve, ofrece un promedio de $21 \mathrm{~cm}$, con máximos de $67 \mathrm{~cm}$ en 73-74 y de $66 \mathrm{~cm}$ durante la ya varias veces mencionada temporada 71-72; que contrastan con el mínimo de sólo $4 \mathrm{~cm}$ en la excepcionalmente anómala (en este caso por ausencia casi total de nieve) de la también ya mencionada 89-90. La variabilidad interanual alcanza un muy elevado $68 \%$.

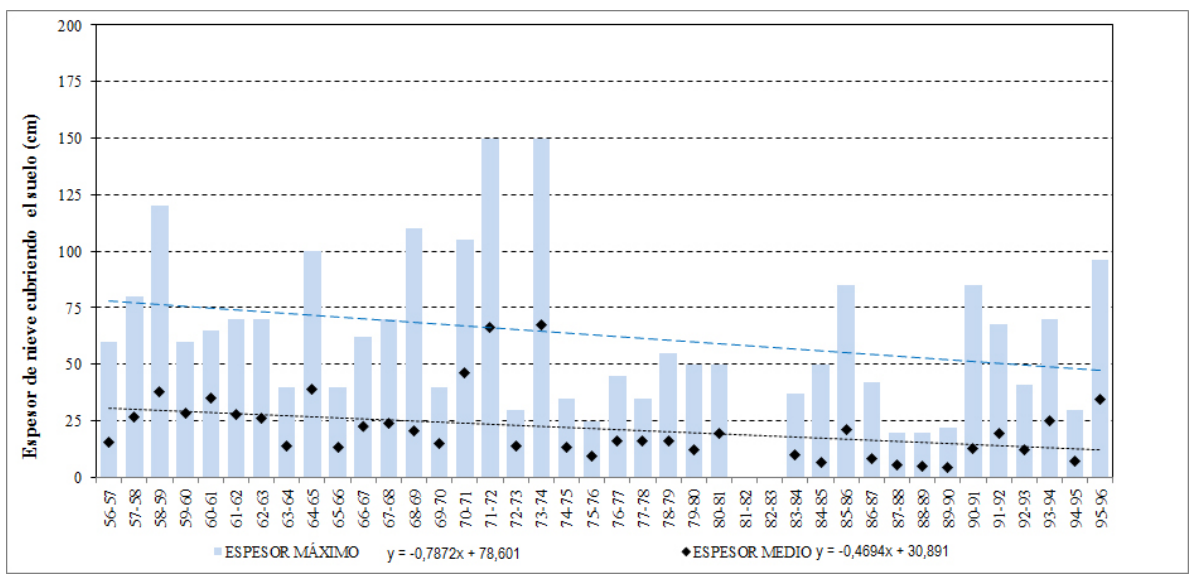

Fig. 6: Evolución de espesores de nieve en La Molina. Periodo 1956-57 a 1995-96.

\section{DISCUSIÓN Y CONCLUSIONES}

El análisis nivométrico realizado para la cota $1.703 \mathrm{~m}$ de La Molina, muestra fundamentalmente dos características principales: por un la lado, el valor modesto de sus índices de nivosidad e innivación, en relación a otras localidades centropirenaicas o de mayor altitud (Cuadrat Prats, 1983; Vilar Bonet y Salvador Franch, 1996; Capel Molina, 2000) y, por otro, sobretodo, la muy fuerte irregularidad en todos los indicadores utilizados, fruto sin duda de la cercana influencia mediterránea. En relación con otras aproximaciones anteriores, habitualmente basadas en el número de días de precipitación en forma de nieve (Xercavins, 1981, 1983), los valores ahora calculados son a grandes rasgos semejantes aunque la disparidad de series utilizadas hace difícil su comparación.

Respecto a los valores nivométricos obtenidos en Núria (Salvador Franch et al., 2014), contrariamente a lo esperado, los índices nivales de La Molina son ligeramente inferiores a los de Núria, a pesar de la posición y orientación supuestamente desfavorables de esta última localidad, debiendo atribuirse esto a la diferencia de altitud y a las series temporales escasamente coincidentes entre ambas.

Todas las variables nivométricas ahora obtenidas para la serie de La Molina (entre 1956 y 1996), presentan rectas de tendencia con una marcada disminución de sus va- 
lores (nivosidad e innivación) (figuras 4, 5 y 6), a la vez que para ese mismo periodo las temperaturas, tanto anuales como invernales, presentan clara tendencia al alza (figura 2). Durante el periodo de estudio, se constata la existencia de una evidente correlación negativa, estadísticamente significativa al 99\% del nivel de confianza, entre las temperaturas medias de noviembre-mayo y la cantidad de nieve caída $(\mathrm{r}=-0,71)$ y también el número de días de precipitación de nieve $(\mathrm{r}=-0,73)$.

En los escasos 6 años coincidentes entre la serie de LM-Font Canaleta (1.703 m) y la de LM-Costa Rasa $(2.050 \mathrm{~m})$, se constata que el incremento de altitud de la segunda supone un aumento del 17\% en la precipitación nival (nivosidad), del $34 \%$ en los días con el suelo cubierto de nieve (innivación) y del 67\% de los días con espesor $\geq$ a $10 \mathrm{~cm}$.

\section{AGRADECIMIENTOS}

Los autores recuerdan con agradecimiento al que fuera durante años observador meteorológico de La Molina, Enric Gubert, por su disposición a facilitar la consulta de los Cuadernos de Observación de La Molina. También a la Estació de Muntanya de La Molina de Ferrocarrils de la Generalitat de Catalunya por darnos acceso a los datos nivometeorológicos de sus archivos. Este trabajo se inscribe dentro de las actividades del Grupo de Investigación consolidado SGR2014-0373 Paisatge i paleoambients a la muntanya mediterrània (Generalitat de Catalunya).

\section{REFERENCIAS}

Capel Molina, J.J. (2000). La nieve y su distribución espacial en la Península Ibérica. Nimbus, 5-6, pp.6-12.

Cuadrat Prats, J.M. (1983). Régimen de días nieve en el Pirineo central y sus factores. Ponencias y Comunicaciones VII Coloquio de Geografía (Pamplona, 1981). Vol. 1, Salamanca: AGE. pp. 65-67.

García Sellés, C. y Salvador Franch, F. (1994). Snowfall analysis in the Eastern Pyrenees. Annalen der Meteorologie, 30, pp. 303-306.

Guilera, J.M. (1931). Carnet d'un esquiador (1915-1930). Barcelona: Llib. Catalonia, 246p.

Martín Vide, J. (1985). Estacionalidad de la precipitación y mediterraneidad en el Pirineo catalán. Notes de Geografia Física, 13-14, pp. 57-65.

Roger, G. (2006). La vall de La Molina. Aproximació històrica. El Mirador. 107p.

Salvador Franch, F. (1985). Aportación al estudio nivoclimático del Pirineo oriental. Notes de Geografia Física, 13-14, pp. 67-84.

Salvador Franch, F., Salvà Villoslada, G., Vilar Bonet, F. y García Sellés, C. (2014). Nivometría y perfiles de innivación en Núria (1.970 m, Pirineo Oriental): $1985-$ 2013. En S. Fernández-Montes y F.S. Rodrigo (Eds.): Cambio Climático y Cambio Global (IX Congreso de la AEC, Almería, 2014). AEC, pp. 729-738.

SMC (2008). Atlas climàtic de Catalunya: periode 1961-1990. Termopluviometria. Barcelona: ICC.

Vila, P. (1926). La Cerdanya. Barcelona: Ed. Barcino, 264p. 
Vilar Bonet, F. y Salvador Franch, F. (1996). Variaciones y tendencia secular de la precipitación de nieve en Andorra (Pirineo Oriental). En M.V. Marzol, P. Dorta y P. Valladares (Eds.): Clima y agua: la gestión de un recurso climático (III Reunión Nac. de Climatología, La Laguna, 1996). La Laguna: AGE, pp. 87-97.

Vilar Bonet, F. y Salvador Franch, F. (2001). Caracterización de episodios de nevadas intensas en el Pirineo oriental catalán. En A.J. Pérez Cueva, E. López Baeza y J. Tamayo Carmona (Eds.): El Tiempo del Clima (II Congreso de la AEC, València, 2001). AEC, pp. 423-434.

Xercavins Comas, A. (1981). Los climas de montaña media y alta en el Pirineo Oriental. Análisis de las precipitaciones. Tesis Doctoral. Universidad de Barcelona.

Xercavins Comas, A. (1983). El régimen anual de nieves en el Pirineo oriental. En Ponencias y Comunicaciones VII Coloquio de Geografia (Pamplona, 1981). Vol. 1, Salamanca: AGE, pp. 69-74. 